\title{
Shifts in the perceived location of a blurred edge increase with contrast
}

\author{
P. J. BEX and G. K. EDGAR \\ University of Wales, College of Cardiff, Cardiff, Wales
}

\begin{abstract}
Perceived brightness is nonlinearly related to luminance. Consequently, any mechanism operating on the (transformed) luminance profile of a blurred edge to detect its location should make errors, and the magnitude of these errors should increase with contrast. The perceived location of a blurred edge was measured at a range of contrasts and a range of blur space constants in a vernier alignment task. It was found that the perceived location of a blurred edge was affected by the contrast and the blur space constant of the edge. At low contrasts, the apparent location of the blurred edge was near the calculated location of the edge, assuming the linear transduction of luminance. At higher contrasts, the perceived location of a blurred edge was shifted toward the dark side of the edge, and the shift increased with contrast.
\end{abstract}

The perceived location of a blurred edge has been modeled using a number of different approaches (Marr \& Hildreth, 1980; Morrone \& Burr, 1988; Watt \& Morgan, 1983). Despite computational differences among these models, all models predict that the apparent location of a symmetrical blurred edge will be at approximately the same point. Marr and Hildreth (1980) suggest that this point occurs at the maximum gradient change (the zero crossing in the second derivative of the retinal image). However, positional uncertainty increases with blur, although the zero crossing remains in the same location. Watt and Morgan (1983) propose an alternative model (MIRAGE), in which the location of a blurred edge is defined by the zero-bounded regions (ZBRs) in the summed outputs of a set of spatial filters. The model predicts the increased positional uncertainty for blurred edges but no change in perceived location. Morrone and Burr (1988) suggest that edges are located at the point where the Fourier components are in phase. For a symmetrical blurred edge, this point corresponds to the center of the edge profile at same location as the zero crossing.

Mather and Morgan (1986) measured the perceived location of a pair of edges in a vernier alignment task. Both edges were at $80 \%$ contrast, and the blur functions (the space constant of an integrated Gaussian) of the edges were varied. In one condition, both edges had the same contrast polarity. In a second condition, the edges had opposite contrast polarity. It was found that when contrast polarity was the same, the edges were aligned such that their zero crossings were aligned. However, when the contrast polarity was opposite, the edges were aligned such that their zero crossings were not aligned and the edges overlapped

This research was supported by SERC and by British Aerospace PLC G. K. Edgar's mailing address is Human Factors Department, Sowerby Research Centre, British Aerospace PLC, FPC 267, P.O. Box 5, Filton, Bristol BS12 7QW, U.K. Correspondence regarding this article should be addressed to P. J. Bex, McGill Vision Research, McGill University, 687 Pine Ave. West, Rm. H4-14, Montreal, PQ, Canada H3A 1 Al. (i.e., there was a shift in the perceived location of the edges toward the dark side of the edge). The results were interpreted as evidence for a nonlinear transformation of the luminance profile of an edge preceding the computation of its spatial position. This shift is anticipated for any of the models of edge location as long as the luminance nonlinearity precedes the localization of the edge.

Naiman and Makous (1993) have recently reported a shift in the apparent location of an edge with the introduction of a narrow, gray strip between the light and dark phases of a sharp edge. Using a vernier alignment task, these authors found that the apparent position of the edge with the gray strip shifted as the luminance of the strip was varied, even if the strip was not visible. The experimental data were well fitted by a simple model involving a nonlinear transformation of the optical signal.

Mather and Morgan (1986) compared the position of a blurred edge of one blur space constant (1.25 arcmin) with that of a sharp edge only when the edges were static and with either the same or the opposite contrast polarity. In this condition, the authors reported a spatial shift of the apparent location of the blurred edge into its dark phase only when contrast polarity was opposite. When the contrast polarity of the edges was the same, the apparent location of the blurred edge was near the location of the zero crossing, although one of their observers (G.M.) did show a slight shift into the dark phase of the blurred edge. A shift into the dark phase of the apparent location of the edge should have been present when the contrast polarity was the same, since the profile of the blurred edge was misrepresented by a luminance nonlinearity. However, Mather and Morgan's design did not measure this because, in most of their conditions, when contrast polarity was the same, both edges were blurred and subject to such a spatial shift.

The power function relating luminance to perceived brightness (Stevens \& Galanter, 1957) has been modeled by Makous, Williams, and MacLeod (1985) with the equation: 


$$
R=1.712 I-0.712 I^{2}
$$

where $R$ is response and $I$ is intensity. The effect of this nonlinearity on the luminance profile of a blurred edge is illustrated in Figure 1, where $I$ has been normalized to span the range 0 to 1 at maximum contrast. It can be seen that the neural representation of the luminance profile of a blurred edge is affected by contrast. It was hypothesized that the profile of a blurred edge would be more veridically represented at low contrast. Correspondingly, at low contrast, the perceived location of a blurred edge would be nearer the calculated location of the edge (zero crossing, $\mathrm{ZBR}$, or phase coincidence), and, at high contrast, the perceived location of a blurred edge would shift toward the

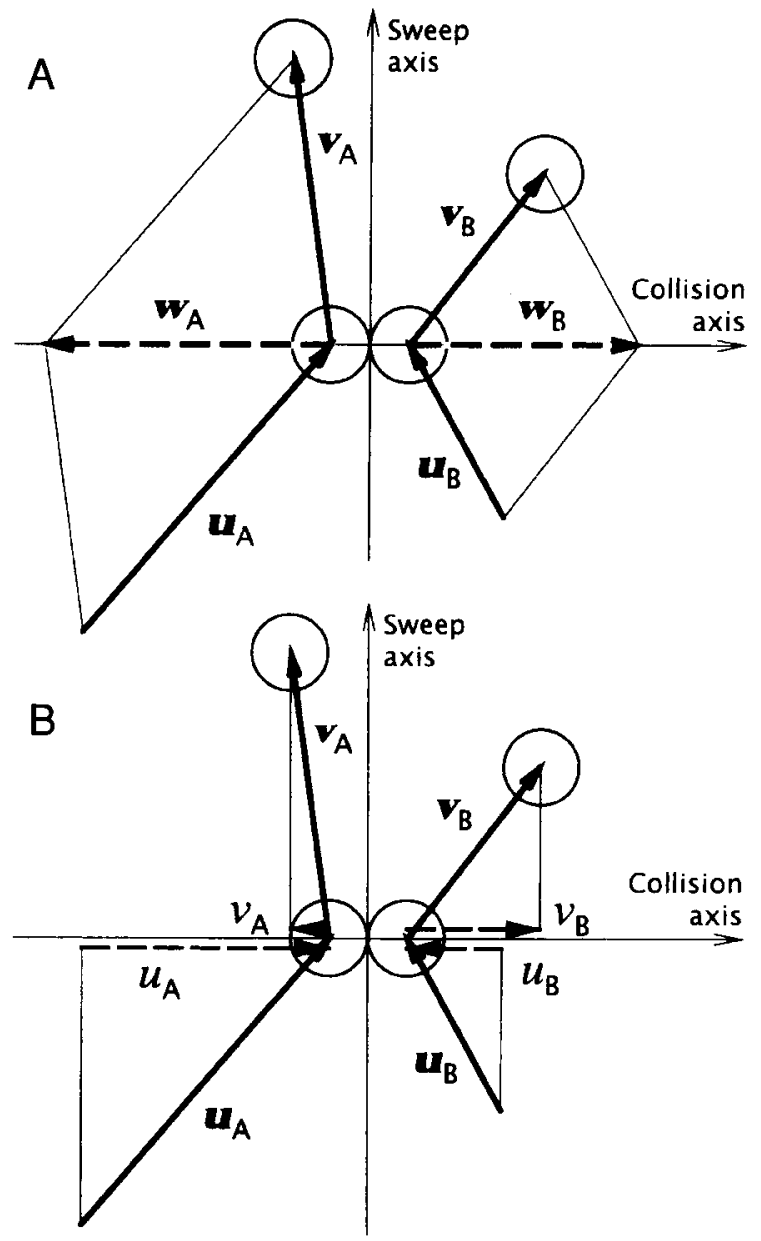

Figure 1. The effect of a luminance nonlinearity on the luminance profile of blurred edges: (A) a $50 \%$ contrast edge, (B) a $10 \%$ contrast edge (note that the axes have been rescaled for clarity). The solid curves show the luminance profiles of half-cycle cosinusoidal, blurred edges. The broken curves show the neural representation of the profile after a luminance nonlinearity (see text). The average luminance component of the change in the profile has been removed for clarity. The vertical broken lines indicate locations of the zero crossings of each profile, the leftmost line in each case represents the zero crossing of original edge, and the rightmost represents the location of the zero crossing of the nonlinear representation of the edge. The location of the zero crossing is shifted toward the dark side of the edge, and the shift increases with contrast. dark side of the edge. Therefore, the position of a static, blurred edge was measured in a vernier alignment task with a static, sharp edge at each of a range of contrasts.

\section{METHOD}

\section{Apparatus}

Stimuli were generated by an image generator (Millipede VR 1000) under the control of a PC microcomputer and were presented on a Hewlett-Packard 1332A X-Y display with white P4 phosphor using a raster technique at a $122-\mathrm{Hz}$ frame rate. The monitor was calibrated carefully, and the image was gamma-corrected using a look-up table. The screen was masked to provide two rectangular windows $\left(2.5^{\circ}\right.$ vertically $\times 4^{\circ}$ horizontally), one above the other, separated by a dark, horizontal strip $\left(0.25^{\circ}\right)$ with a bright central fixation spot. The screen was viewed from a distance of $1.14 \mathrm{~m}$.

\section{Stimuli}

The stimuli were a vertical sharp edge (standard) and a vertical blurred edge (match), the contrasts of which were manipulated. Contrast was measured as the Michelson contrast: $\left(\mathrm{L}_{\max }-\mathrm{L}_{\min }\right)$ / $\left(\mathrm{L}_{\max }+\mathrm{L}_{\min }\right)$. The sharp edge was simply a step function in luminance. The blurred edge was created using half a cycle of cosinusoidal luminance variation in place of the step (see Figure 1). The extent of blur was varied by changing the spatial period of the cosine function. The blur space constant of the edge was defined as the width of the cosinusoidal blurring function and corresponded to one half cycle of the cosine function. The blur space constant of the match edge was set at the beginning of each run at 0 (sharp edge), 15 , or 30 arcmin. The two edges were presented simultaneously -one in the upper window, the other in the lower window.

\section{Procedure}

The subject was seated at the required viewing distance and instructed to fixate the small spot in the center of the screen. Before the start of the experiment, each window was a homogenous mean luminance field for $1 \mathrm{~min}$ to ensure a constant state of adaptation. At the end of $1 \mathrm{~min}$, a tone sounded to signal the start of the experimental run. The subject was instructed to press either of the two response buttons when ready, which initiated the run. Two seconds after the buttonpress, the blurred match edge and the sharp standard edge appeared. The position of the pair of edges was subject to a small random spatial shift $( \pm 0-10$ arcmin $)$ between trials to reduce the effects of afterimages. The contrasts of the edges were equal and were set at the beginning of the trial. The particular window in which either edge was presented was randomized between trials, except when the match edge was sharp ( 0 arcmin blur function). On these trials, the match edge was presented in the upper window for two runs and in the lower window for two runs to minimize hemifield differences. The relative positions of the edges were set between trials according to a method of constant stimuli. There were five spatial offsets, and each was presented 10 times in random order. After $500 \mathrm{msec}$, the edges were replaced by blank mean luminance fields. The subjects were required to respond, by pressing one of two console buttons, in which direction the standard edge was offset with respect to the match edge. When the match edge was also sharp $(0$ arcmin blur function), the subjects were informed of the window in which the match edge was presented and were required to indicate the offset of the standard edge with respect to the edge in this window. After the subject had responded, there followed a 2-sec intertrial interval in which a blank mean luminance field was displayed. This was followed by the next trial. Each run consisted of 50 trials.

Match edge position (relative to the standard) was defined as the spatial offset at which the subject reported seeing the match edge offset in one direction on $50 \%$ of trials for each blur space constant at each contrast. The $50 \%$ point was inferred by fitting a psychometric function (Weibull, 1951) to the data. There were 50 trials per run, 
four runs per subject, and 2 subjects (one of the authors and a naive observer) for all conditions. The order in which the different blur functions and contrasts were tested was randomized for each subject.

\section{RESULTS AND DISCUSSION}

The spatial offset at which the two edges appeared to be in the same location with a probability of $50 \%$ is shown for each subject in Figure 2. The apparent position of a static, high-contrast, blurred edge is not at the location of the zero crossing, ZBR, or phase alignment, calculated assuming a linear transduction of luminance. At high contrasts, there is a shift in the apparent position of the blurred edge to-
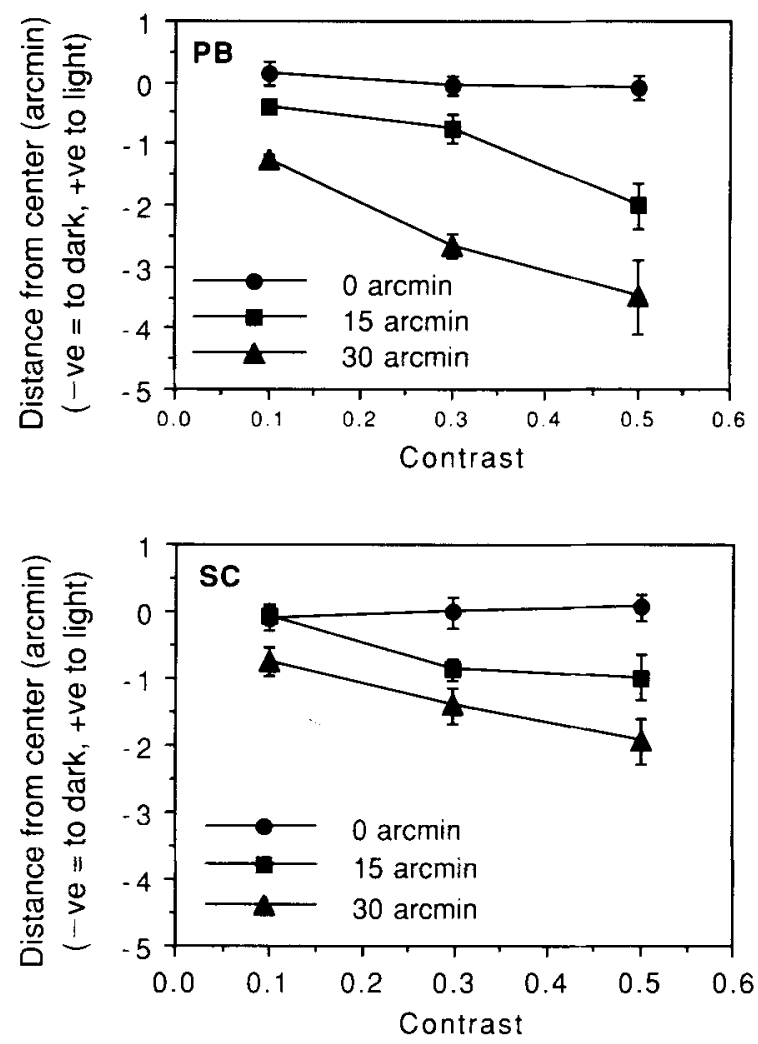

Figure 2. The perceived location of a blurred edge measured using a vernier alignment task for 2 observers. The apparent location of a sharp edge was compared with that of a blurred edge, presented one above the other. The blur space constant is shown in the key. Data show the point at which the subjects reported equal position of both edges with a probability of $50 \%$. Negative distances indicate a shift in the apparent location of the blurred edge into its dark phase, and positive values indicate a shift into the bright phase. The location of the blurred edge is given in terms of the distance from the center of the blur space constant, which is equivalent to the calculated position of the edge. Error bars represent $\pm 1 S E$. ward the dark side of the edge. The magnitude of the apparent shift increases with the blur and contrast of the edge. As contrast decreases, the apparent position of a blurred edge shifts toward the center of the luminance profile, where the position of the edge is predicted by models of edge localization, as described above. The apparent location of sharp edges ( 0 arcmin blur function) is unaffected by contrast. The general increase in the error bars as the blur of the match edge increases is consistent with increases in positional uncertainty reported for blurred edges (Watt \& Morgan, 1983).

The increase in the shift of the apparent spatial position of a blurred edge with the extent of blur is consistent with a nonlinear stage of luminance transduction preceding the localization of an edge. The nonlinearity increases with contrast, and the accuracy of the neural representation of a blurred edge is inversely related to contrast. The apparent position of a blurred edge is therefore closer to the zero crossing (Marr \& Hildreth, 1980), the ZBR (Watt \& Morgan, 1985) or to the point at which phases coincide (Morrone \& Burr, 1988) at lower contrasts. The present data provide further evidence that a luminance nonlinearity precedes the operations involved in the identification of spatial primitives in human vision. The reliable differences between subjects suggest that although a saturating nonlinearity can describe the relationship between luminance and perceived brightness qualitatively, it is unlikely that a general function will be able to model the nonlinearity quantitatively for more than 1 subject.

\section{REFERENCES}

Makous, W., Williams, D. R., \& Macleod, D. I. A. (1985). Nonlinear transformation in human vision. Journal of the Optical Society of America $A, 13, \mathrm{P} 80$.

Marr, D., \& Hildreth, E. (1980). Theory of edge detection. Proceedings of the Royal Society of London: Series B, 207, 187-217.

Mather, G., \& Morgan, M. (1986). Irradiation: Implications for theories of edge localisation. Vision Research, 26, 1007-1015.

Morrone, C., \& BURr, D. (1988). Feature detection in human vision: A phase-dependent energy model. Proceedings of the Royal Society of London: Series B, 235, 221-245.

Naiman, A. C., \& Makous, W. (1993). Undetected grey strips displace perceived edges non-linearly. Journal of the Optical Society of America $A, 10,794-803$.

Stevens, S. S., \& Gal.anter, E. M. (1957). Ratio scales and category scales for a dozen perceptual continua. Journal of Experimental Psychology, 54, 377-411.

WATt, R. J., \& Morgan, M. (1983). The recognition and representation of edge blur: Evidence for spatial primitives in vision. Vision Research, 23, 1465-1477

WATt, R. J., \& MORGan, M. (1985). A theory of the primitive spatial code in human vision. Vision Research, 25, 1661-1674.

WeIBULl, W. (1951). A statistical distribution function of wide applicability. Joumal of Applied Mathematics, 18, 292-297.

(Manuscript received August 22, 1994; revision accepted for publication April 18, 1995.) 